University of Wollongong

Research Online

Faculty of Engineering and Information

Faculty of Engineering and Information

Sciences - Papers: Part A

Sciences

$1-1-2014$

\title{
An effective localization method for robotic endoscopic capsules using multiple positron emission markers
}

Trung Duc Than

University of Wollongong, dtt581@uowmail.edu.au

Gursel Alici

University of Wollongong, gursel@uow.edu.au

Steven Harvey

University of Wollongong, sharvey@uow.edu.au

Graeme O'Keefe

University of Melbourne, Graeme.OKEEFE@austin.org.au

Hao Zhou

University of Wollongong, hz467@uowmail.edu.au

See next page for additional authors

Follow this and additional works at: https://ro.uow.edu.au/eispapers

Part of the Engineering Commons, and the Science and Technology Studies Commons

Research Online is the open access institutional repository for the University of Wollongong. For further information contact the UOW Library: research-pubs@uow.edu.au 


\title{
An effective localization method for robotic endoscopic capsules using multiple positron emission markers
}

\begin{abstract}
The wireless capsule endoscope (WCE) is a first-line medical tool for the diagnosis of many gastrointestinal (GI) tract diseases such as obscure GI bleeding, Crohn's disease, small bowel tumors, and Celiac disease. Over the past few years, significant research attention has been paid to upgrading the WCE from a diagnostic-only tool to an active medical robot having not only diagnostic capabilities but therapeutic functionalities, such as biopsy, microsurgery, and targeted drug delivery, as well. One of the major limitations that impedes the development of such a robotic-type endoscope is the lack of a highly accurate localization system. In this paper, we present an experimental evaluation of a new real-time localization method (patent pending) based on tracking three positron emission markers embedded in the cover of an endoscopic capsule. Coincidence gamma rays emitted from the markers are detected by surrounding gamma ray detectors. The position and orientation information of the capsule can then be extracted by an effective tracking algorithm. The experiments were conducted in two different commercial positron emission technology (PET) scanners: Philips Allegro and Philips TF64. The experimental results show that the proposed localization method could provide less than $0.5-\mathrm{mm}$ position error and $2.4^{\circ}$ orientation error in a localization time interval of $50 \mathrm{~ms}$ with an average computational time of $6 \mathrm{~ms}$ per time interval. Zero power consumption and zero space occupation inside the capsule are additional advantages of this localization method.
\end{abstract}

\section{Keywords}

endoscopic, capsules, multiple, markers, localization, positron, effective, emission, method, robotic

\section{Disciplines}

Engineering | Science and Technology Studies

\section{Publication Details}

Than, T. Duc., Alici, G., Harvey, S., O'Keefe, G., Zhou, H., Li, W., Cook, T. \& Alam-Fotias, S. (2014). An effective localization method for robotic endoscopic capsules using multiple positron emission markers. IEEE Transactions on Robotics, 30 (5), 1174-1186.

\section{Authors}

Trung Duc Than, Gursel Alici, Steven Harvey, Graeme O'Keefe, Hao Zhou, Weihua Li, Trent Cook, and Sharon Alam-Fotias 


\title{
An effective localization method for robotic endoscopic capsules using multiple positron emission markers
}

\author{
Trung Duc Than, Gursel Alici*, Steven Harvey, Graeme O’Keefe, Hao Zhou, Weihua Li, Trent Cook, \\ and Sharon Alam-Fotias
}

\begin{abstract}
Wireless capsule endoscope (WCE) is a first-line medical tool for the diagnosis of many gastrointestinal (GI) tract diseases such as obscure gastrointestinal bleeding, Crohn's disease, small bower tumors, and Celiac disease. In the past few years, significant research attention has been attracted to upgrade the WCE from a diagnostic-only tool to an active medical robot having not only diagnostic capabilities but also therapeutic functionalities such as biopsy, microsurgery, and targeted drug delivery. One of the major limitations that impedes the development of such a robotic-type endoscope is the lack of a highly accurate localization system. In this paper, we present an experimental evaluation of a new real-time localization method (patent pending) based on tracking three positron emission markers embedded in the cover of an endoscopic capsule. Coincidence gamma rays emitted from the markers are detected by surrounding gamma ray detectors. The position and orientation information of the capsule can then be extracted by an effective tracking algorithm. The experiments were conducted in two different commercial PET scanners: Philips Allegro and Philips TF64. The experimental results show that the proposed localization method could provide less than $0.5 \mathrm{~mm}$ position error, and $2.4^{\circ}$ orientation error in a localization time interval of $50 \mathrm{~ms}$ with an average computational time of $6 \mathrm{~ms}$ per time interval. Zero power consumption and zero space occupation inside the capsule are also advantages of this localization method.
\end{abstract}

Index Terms-Localization, microrobots, tracking, wireless capsule endoscopes, positron emission markers.

\section{INTRODUCTION}

$\mathbf{I}^{\mathrm{N}}$ $\mathrm{N}$ being able to explore the entire gastrointestinal (GI) tract comfortably and safely, Wireless Capsule Endoscope (WCE) has been widely used as a preferred tool for the

This work was supported in part by the Intelligent Nano-Tera Research Systems Laboratory. Asterisk indicates corresponding author:

T. D. Than, H. Zhou, and W. Li are with the School of Mechanical, Materials and Mechatronic Engineering, University of Wollongong, N.S.W. 2522, Australia (e-mail: dtt581@uowmail.edu.au; hz467@uowmail.edu.au; weihuali@uow.edu.au).

*G. Alici is with the School of Mechanical, Materials and Mechatronic Engineering, and the ARC Centre of Excellence for Electromaterials Science, University of Wollongong, N.S.W. 2522, Australia (e-mail: gursel@uow.edu.au)

S. Harvey is with the Department of Nuclear Medicine, Wollongong Hospital, Wollongong, N.S.W. 2500, Australia (e-mail: Steven.Harvey@sesiahs.health.nsw.gov.au)

G. O'Keefe is with the Department of Nuclear Medicine and Centre for PET, University of Melbourne, Austin Health, Victoria, 3084, Australia (email: Graeme.O'KEEFE@austin.org.au)

T. Cook and S. Alam-Fotias are with the Department of Nuclear Medicine, Nepean Hospital, Nepean, N.S.W 2751, Australia (e-mail: Trent.Cook@swahs.health.nsw.gov.au; Sharon.AlamFotias@swahs.health.nsw.gov.au)

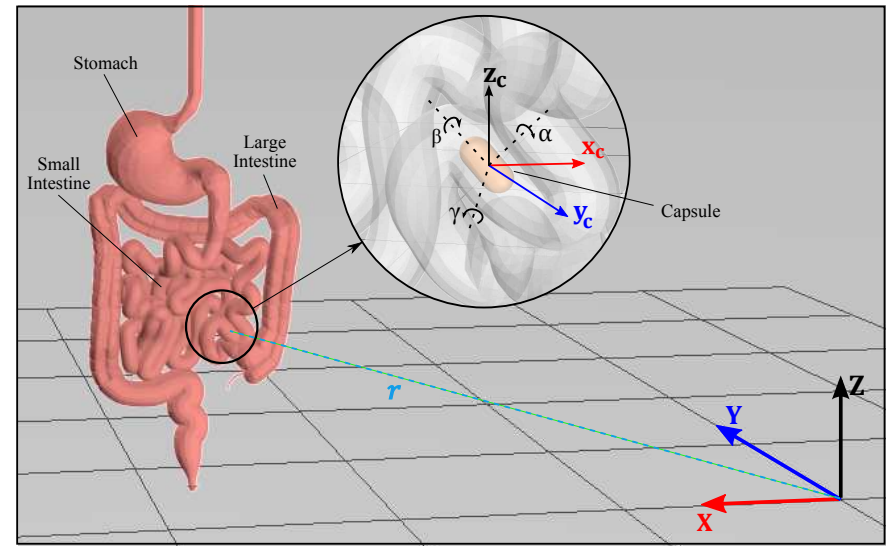

Fig. 1. Position information $\left(x_{c}, y_{c}, z_{c}\right)$ and orientation information $(\alpha, \beta$, $\gamma$ ) of a capsule inside a GI tract with respect to a reference coordinate system $(\mathrm{X}, \mathrm{Y}, \mathrm{Z})$

diagnosis of many diseases in the GI tract. To date, more than two million endoscopic capsules have been used all over the world [1] since the introduction of the first WCE in 2000 [2]. WCE technology has been gaining substantial research attention recently, seen by the impressive number of studies on different aspects of WCE that have been published worldwide in the last ten years [3]. Current ongoing research studies are focusing on upgrading the endoscopic capsule to a more powerful medical device that can carry out both diagnostic and therapeutic capabilities [4], [5] such as biopsy [6], microsurgery and targeted drug delivery [7], [8].

Developing a fully robotic WCE is a challenging task due to two major limitations of the current commercial WCE. Firstly, the capsule is unable to be accurately located when it travels along the GI tract. Therefore, although lesions can be detected by reviewing endoscopic images of abnormalities in the GI tract, their exact locations are unknown. Without a localization system, other important information for the diagnosis such as the distance that the capsule has traveled or the region of the GI tract in which the capsule is located is also missing or very difficult to estimate. This limits the diagnostic efficacy of WCE. More importantly, lack of the position and orientation data of the capsule also constrains the capability to return to the sites of interest for re-inspection or follow-up interventions such as drug delivery or surgical operations. Another major limitation of WCE is that the capsule moves unpredictably and 
passively through the GI tract by means of natural peristalsis and gravitational effects. Therefore, the capsule is unable to stop, to turn or accelerate, resulting in potential issues such as missing symptoms or capsule retention. In addition, it is impossible to perform a biopsy or drug delivery when the capsule movement is uncontrollable. Active locomotion is thus a vital requirement for the next generation of WCE. This again emphasizes the necessity of having an accurate localization system for WCE as localization is essential to provide a prompt feedback in any position/orientation control systems. Figure 1 shows localization data of an endoscopic capsule in a GI tract with respect to a reference coordinate system.

As reported in the literature, various techniques have been proposed to overcome the localization problem, yet so far none of them satisfy one or more of the following mandatory requirements: high accuracy, safety, real-time tracking, compatibility with magnetic actuation systems, and low-power consumption [9].

Measuring the strength of radio-frequency (RF) signals transmitted from the capsule to antennas placed on the patient's abdomen for the transmission of captured images was the first technique proposed for localization [10]. This method only provides $2 \mathrm{D}$ tracking with large position error of $37.7 \mathrm{~mm}$. Several efforts have been made to improve the RF based method [8], [11], [12], however high accuracy is difficult to achieve due to the complex RF absorption properties of inbody tissues and organs.

Recently, the most active area of research in solving the localization problem for WCE has been magnetic localization [9]. The proposed methods can be classified into two main groups. The first group utilizes external magnetic sensors to localize a permanent magnet integrated inside a capsule [13], [14]. Although this approach is safe and can achieve reasonable accuracy, potential of interference with magnetic actuation systems is a limitation. This limitation is significant as magnetic actuation is anticipated to be a future approach for automatic control of capsule movement [15], [16]. Although time-multiplexed sensing and actuation has been suggested to overcome the interference problem [17], this solution would not ensure real-time tracking and prompt feedback for the actuation [9]. Conversely, in the second group, magnetic sensors are placed inside the capsule to measure the external magnetic field which is generated for maneuvering purposes [18]-[22]. In these systems, there appears no interference between magnetic sensing and magnetic driving. However, one common drawback of this approach is low compatibility with other actuation systems where a different approach for active movement control is used [21]. More importantly, magnetic sensors inserted inside the capsule would occupy significantly more capsule space and consume extra battery power, already needed for other integrated functions of WCE [23].

Other localization methods include computer vision [24], ultrasound [25], [26], and MRI [27], which have some potential drawbacks. In the computer vision method, the tracking performance largely depends on the image capturing rate and the speed of the capsule. On the other hand, using ultrasound for WCE localization would be a challenge due to the acoustic

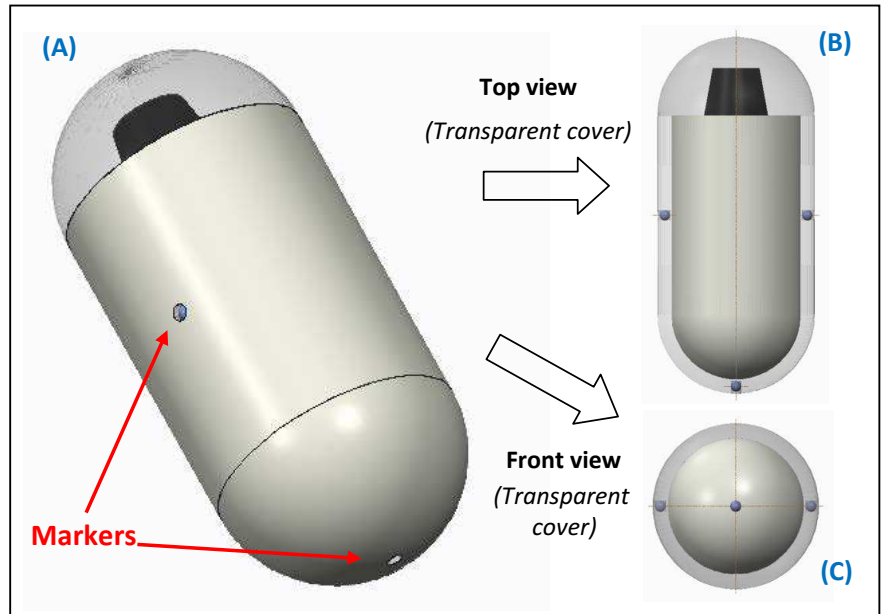

Fig. 2. Conceptual design of a PECapsule. (A): Three positron emission markers are embedded in the cover of the capsule. (B) and (C): Top and front views (cover is drawn transparent for better visualization of the markers). (Note: figure not drawn to scale).

impedance mismatch [15]. Although MRI could enable accurate capsule tracking, the high cost and the possibility of having the interference between the sensing part and magnetic actuation would be a disadvantage.

We have proposed a novel localization method based on tracking multiple positron emission markers embedded in the cover of an endoscopic capsule [28]. Using simulation data obtained from the GATE (Geant4 Application for Emission Tomography by OpenGATE Collaboration) simulation toolkit, we showed that the proposed method could provide realtime tracking with an average position error of approximately $0.4 \mathrm{~mm}$ and an average orientation error of less than $2^{\circ}$. This localization method, which is expected to be compatible with any other actuation systems, does not occupy any space inside the capsule or consume any power from the built-in battery. The contribution of this study is to experimentally evaluate and quantify the performance of the localization method (i.e. determining the position and orientation of a capsule endoscope accurately). The experiments were conducted in two different commercial PET scanners: Philips TF64 and Philips Allegro (Philips Medical Systems, Cleveland, USA). To the best of our knowledge, the evaluation results obtained in this paper has shown better localization performance than other localization methods which have been presented in the literature [9].

In Section II, the overview of the localization method and the tracking algorithm is summarized. Section III presents the experimental design and setup. Section IV shows the experimental results and performance evaluation of the localization method. Discussion and Conclusion are presented in Section V and Section VI, respectively.

\section{OVERVIEW OF THE LOCALIZATION METHOD}

\section{A. Principle of operation}

In order to localize a WCE, three positron emission markers are embedded in the cover of the capsule, as shown in Fig. 2. Due to annihilations of emitted positrons from the 


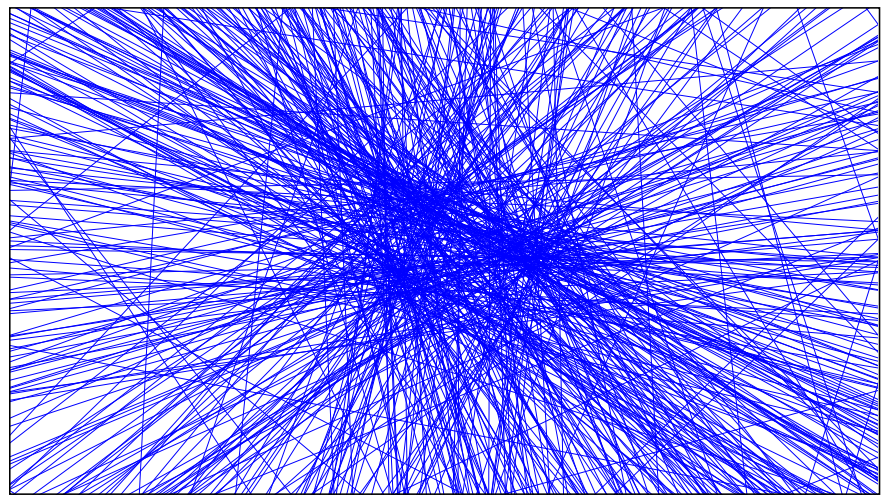

Fig. 3. Coincidence lines arising from three markers in one localization time interval. The location of the markers is evident.

markers with electrons from the surrounding environment, a number of gamma rays are generated in each localization time interval. These gamma rays can then be detected by gamma ray detectors placed around the patient's body. Each gamma ray detected by the detectors generates a line of response, called a coincidence line. Figure 3 illustrates coincidence lines collected in one localization time interval (or localization run).

These coincidence lines can be used to determine the locations at which the annihilation events occur [29]. Ideally, two non-parallel coincidence lines are able to locate a single positron emission source. However, due to positron range (the distance that the positron has traveled before the annihilation), noncollinearity, scattered coincidence, and random coincidence [30], a large number of coincidence lines are required to ensure accurate tracking. The location of the single source can be estimated by finding the point that minimizes the sum of the squared distance to the coincidence lines. Based on this idea, it is possible to track multiple positron emission sources if the coincidence lines are classified appropriately such that coincidence lines in the same group are assumed to arise from the same source [31]-[33].

\section{B. Conceptual design of a PEcapsule}

We call an endoscopic capsule carrying positron emission markers a PEcapsule. On a $1.5 \mathrm{~mm}$-thick cover of a PEcapsule, there are three $\phi 1 \mathrm{~mm} \times L 1.25 \mathrm{~mm}$ cylindrical holes as shown in Fig. 2 so that the markers can be attached to the PEcapsule before the start of an endoscopy procedure. A $0.25 \mathrm{~mm}$ thick lid at the top of the hole will then lock the marker to prevent it from being released during the procedure. The three markers form a triangle with sides of $10 \mathrm{~mm}, 13 \mathrm{~mm}$ and $13 \mathrm{~mm}$ long respectively.

As explained in [28], any positron emission isotope with a half-life of more than a few hours can be considered to be a suitable radioactive source for the localization of WCE. We chose ${ }^{22} \mathrm{Na}$ due to its high availability, wide use in the field of Positron Emission Tomography (PET) imaging, and low positron range. Moreover, the long half-life of ${ }^{22} \mathrm{Na}(2.6$ years) would allow future re-uses of the markers without a frequent need for replenishment. Each marker has a $\phi 1 \mathrm{~mm}$ spherical shape and contains a $\phi 0.5 \mathrm{~mm}$ spherical radioactive core of $50 \mu \mathrm{Ci}(1.85 \mathrm{MBq})^{22} \mathrm{Na}$ at the center, covered in a $0.25 \mathrm{~mm}$-thick acrylic shell.

\section{Tracking algorithm}

We have developed a tracking algorithm based on a Fuzzy C-means clustering algorithm to extract position data of the three markers from a list of coincidence lines collected in each localization run. The algorithm, satisfies both requirements of high accuracy and real-time tracking. By using the following four steps, the tracking algorithm would also be applied to track more than three markers for other applications if necessary.

1) Step 1: The algorithm firstly removes corrupted lines by combining an outlier removal method and iteratively finding the center of the triangle formed by the three markers. This step is essential to generate clean data for the next steps.

2) Step 2: Since the clustering algorithm in Step 4 requires initial values to start, the purpose of this step is to provide good initial estimates of the markers' positions. The initial positions are estimated through finding optimal rotational angles of the triangle when it is rotated around the triangle's center obtained is Step 1. This initialization step is only activated at the start of the localization procedure or when the failure prediction method in Step 3 has identified a potential failure. For other localization runs, the last known positions of the markers are used as initial values.

3) Step 3: In some extreme cases in which the capsule encounters a sudden change in its movement, the prior knowledge of the markers' position may not be reliable to be initial values. To prevent the clustering algorithm in Step 4 from failure in such cases, this step checks and then activates Step 2 again to provide better initial estimates.

4) Step 4: This step, which is a core part of the tracking algorithm, is based on the Fuzzy C-means clustering algorithm [34] to classify the coincidence lines into three groups such that coincidence lines in each group are supposed to be generated from the same marker. The position of each marker can then be determined by finding the point that minimizes the sum of squared distance to all coincidence lines in the corresponding group.

Starting with initial values obtained in Step 2, this step iteratively performs two consecutive tasks until the estimate of markers' positions is converged. These two tasks are:

a) Assign each coincidence line $l_{n}(n=1, \ldots, N)$ with a degree of membership to each cluster $(k=1, \ldots, 3)$ :

$$
u_{n k}^{(i)}=\frac{f_{k}^{(i)} \cdot\left(\frac{1}{\left\|\vec{d}\left(\vec{M}_{k}^{(i)}, l_{n}\right)\right\|^{2}}\right)^{\frac{1}{(q-1)}}}{\sum_{j=1}^{3} f_{j}^{(i)} \cdot\left(\frac{1}{\|{\vec{d}\left(\vec{M}_{j}^{(i)}, l_{n}\right) \|^{2}}^{\frac{1}{(q-1)}}}\right.}
$$

where $\mathrm{N}$ is the number of coincidence lines collected, $i$ is the iteration index, $\left\|\vec{d}\left(\vec{M}_{k}^{(i)}, l_{n}\right)\right\|$ is the distance from marker $\vec{M}_{k}$ to line $l_{n}$, q is a weighting exponent which controls the "fuzziness" of the resulting clusters (it can be any number greater than 1), and $f_{k}$ is relative activity of marker $\vec{M}_{k}$ which 

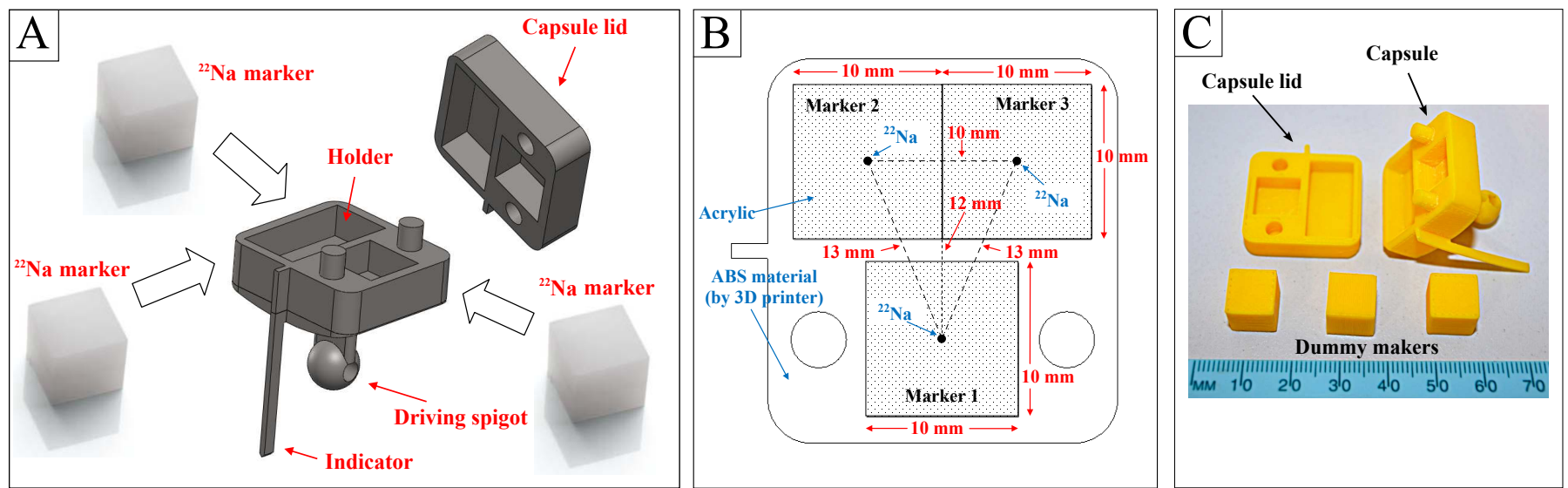

Fig. 4. Design of the experimental capsule and markers. (A): 3D models of the capsule and markers. (B): 2D sketch of the top view of the capsule to show how the markers are arranged. (C): The size of a 3D-printed capsule with "dummy" markers for illustration

indicates how large the size of its corresponding cluster is compared to other clusters. At the first iteration

$$
f_{1}^{(i=0)}=f_{2}^{(i=0)}=f_{3}^{(i=0)}=\frac{1}{3}
$$

b) Update new estimate of the markers' positions and their relative activity:

$$
\vec{M}_{k}^{(i+1)}=\vec{M}_{k}^{(i)}+c \cdot \frac{\sum_{n=1}^{N}\left(u_{n k}^{(i)}\right)^{q} \cdot \vec{d}\left(\vec{M}_{k}^{(i)}, l_{n}\right)}{\sum_{n=1}^{N}\left(u_{n k}^{(i)}\right)^{q}}
$$

where $c$ is a scale constant which controls the iteration speed (its optimal value is chosen to be 1.5)

$$
f_{k}^{(i+1)}=\frac{\sum_{n=1}^{N} u_{n k}^{(i)}}{\sum_{j=1}^{3} \sum_{n=1}^{N} u_{n j}^{(i)}}
$$

A complete explanation of the entire tracking algorithm can be found in [28].

\section{EXPERIMENTS}

\section{A. Experimental apparatus}

In order to prove the concept of the proposed localization method and to evaluate the performance of the tracking algorithm, an experimental apparatus was designed and fabricated by the 3D printing technique. The design of the apparatus needed to satisfy the following criteria:

- A phantom filled with water is essential to imitate the photon attenuation property of a human body. In the field of PET, water phantoms are often used since gamma rays encounter similar attenuation and scattering characteristics when they travel through a patient body and a water phantom.

- The capsule carrying the markers is able to move automatically in both linear and non-linear trajectories with different orientations inside the enclosed water phantom.

- For performance evaluation, there needs to be a way to determine the actual position and orientation of the capsule during the experiments (visually, for example) to compare the actual data with estimated data obtained by the tracking algorithm.

- The testing procedure can be conducted repeatedly multiple times without a need of opening/closing the water phantom for re-assembling or calibrating the internal components in the phantom.

Based on these requirements, the experimental design was as follows

1) Radioactive Markers: In the experiments, three commercial spot markers MMS09 (by Eckert \& Ziegler Isotope Products Inc, US) (Fig. 4A) were used. Each marker contains $0.25 \mathrm{~mm}$-diameter spherical radioactive core of ${ }^{22} \mathrm{Na}$ encapsulated at the centroid of a cubic acrylic cover with a size of 10 x $10 \times 10 \mathrm{~mm}$. The cover is used to seal the active core. The radioactivities of the markers on the day of experiment were measured as $1.60 \mathrm{MBq}, 1.48 \mathrm{MBq}$ and $1.47 \mathrm{MBq}$, respectively.

Although the size of the marker is larger than the conceptual design mentioned in Subsection II-B (due to the acrylic cover), this will not affect the proof-of-concept purpose of this study as well as the feasibility of the proposed method. This is because of the fact that acrylic has very low gamma-ray attenuation coefficient and the two important factors (the size of the radioactive cores and their relative distances) that would impact the tracking performance are made exactly the same as designed in Fig. 2. In addition, the markers can be fabricated with custom designs in practice using similar techniques that have been used to produce commercial brachytherapy seeds [31].

2) Experimental capsule: The tracking algorithm is able to localize not only a static capsule but also a moving one. Therefore, the experimental capsule was equipped with extra components for locomotion, which has not been available in commercial WCEs. The size of the experimental capsule in this study was not critical and thus not necessarily the same as that of commercial WCEs. The capsule has three parts: a driving spigot, a holder, and an indicator as shown in Fig. 4A. The driving spigot was connected with a cable through its center so that the capsule could be fed to move forward or backward. The holder is able to retain the markers firmly, and 


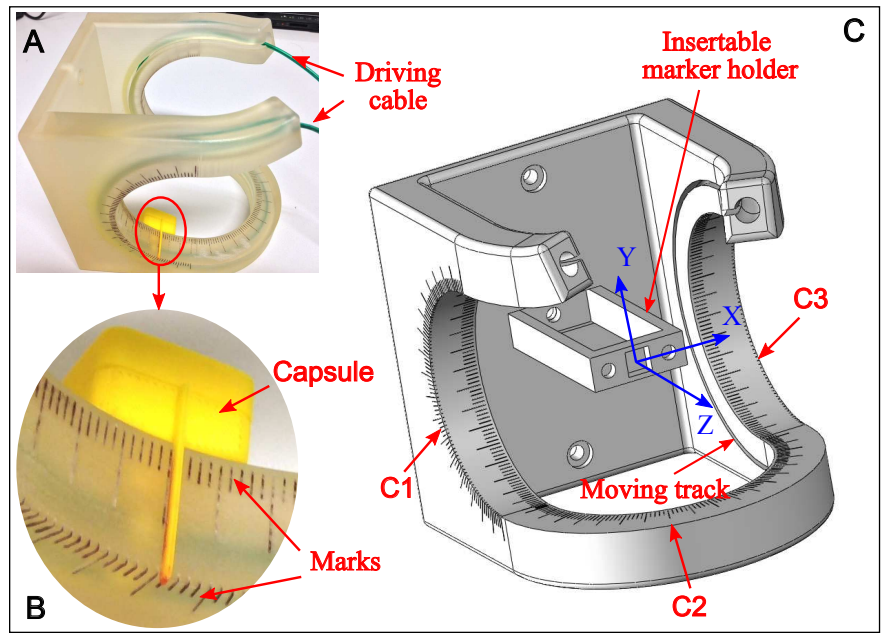

Fig. 5. Design of the moving track. (A): A 3D-printed track along which a capsule attached with a driving cable moves. (B): The capsule is enlarged for clearer view of the indicator and the marks. (C): A 3D design model of the moving track. The track consists of three curves $(\mathrm{C} 1, \mathrm{C} 2$, and $\mathrm{C} 3)$ which are three halves of three circles in $\mathrm{YZ}$ plane, $\mathrm{XZ}$ plane and $\mathrm{YZ}$ plane respectively.

to ensure their relative distances are always fixed at $13 \mathrm{~mm}$, $13 \mathrm{~mm}$ and $10 \mathrm{~mm}$ respectively during the experiments. The indicator measures the position and orientation of the capsule when it moves along a designed track, which is described in more detail below.

3) Moving track: In order to move the capsule along a known trajectory, a moving track was designed and printed by a $17 \mu m$-resolution 3D printer (ProJet 3510 HDPlus Printer, 3D Systems Company) as shown in Fig. 5A. The cable attached to the driving spigot of the capsule was threaded through the entire captive track. By pulling each end of the cable, the capsule could be fed along the track in both directions. The track is composed of three halves of three circles $(\mathrm{C} 1, \mathrm{C} 2$ and $\mathrm{C} 3$ ) with diameters of $116 \mathrm{~mm}$ (in YZ plane), $112 \mathrm{~mm}$ (in XZ plane) and $116 \mathrm{~mm}$ (in $\mathrm{YZ}$ plane) respectively as can be seen in Fig. 5C.

The two ends of the cable were connected to an external pulley system which has three pulleys with teeth as illustrated in Fig. 6. Each end of the cable went through each gap between the middle pulley and the other two pulleys. The middle pulley was driven by a DC motor. As soon as the motor operates, one end of the cable is pulled and the other end follows. A reversible speed controller, which controls the speed of the motor, allows the capsule to move with different speeds in opposite directions.

By having the indicator and marks engraved on the track, the position of the capsule with respect to the track could be visually determined. Three small cameras were placed around the experimental apparatus to capture the movement of the capsule (Fig. 6) allowing the actual position coordinates and orientation of the capsule with respect to a reference coordinate system to be recorded during the experiments.

On the body of the track there was an insertable marker holder (Fig. 5C). This part was only inserted into the track at the beginning of the experiment for calibration which is explained in Subsection III-B.
4) Phantom: We employed a commercial Jaszczak Phantom (Fig. 6), which is widely used in PET studies, for the experiments. The phantom was filled with water to simulate the photon attenuation of a human body. The cable was connected to the pulley system through a hole at the top of the phantom, placed horizontally. To fix the position of the track, it was attached to the bottom of the phantom by plastic screws.

\section{B. Experimental procedure}

Two sets of experiments were conducted on two different PET systems (a Philips Allegro/GEMINI PET system and a Philips TF64/GEMINI PET system) at the Austin Health Hospital, Melbourne, Australia. The experimental apparatus was placed on the patient bed (Fig. 7) and then moved to the centroid of the PET scanner. In each experiment, the apparatus was scanned with 5 different speeds of capsule movement (7.5, 12, 17, 21 and $27 \mathrm{~mm} / \mathrm{s}$ ). The capsule moved from one end to the other end of the track and returned before a new speed was set.

The input of the tracking algorithm is a list of Cartesian coordinates of two ends of coincidence lines. These data are dependent on the position and orientation of a reference coordinate system. For ease of calculation, we chose the centroid of the PET scanner as the origin of the reference coordinate system $\mathrm{x}, \mathrm{y}, \mathrm{z}$ axes of which are the lateral axis, vertical axis and longitudinal axis, respectively.

In order to determine the actual position and orientation of the capsule with respect to the reference coordinate system, the local coordinate system of the track needs to be aligned with the reference coordinate system. This could be done by manually maneuvering the position of the patient bed with the aid of laser lights from the built-in alignment system. However, the centroid of the PET scanner could only be located roughly within a few millimeters of accuracy by the alignment system. Therefore, an extra step (calibration step) was created before

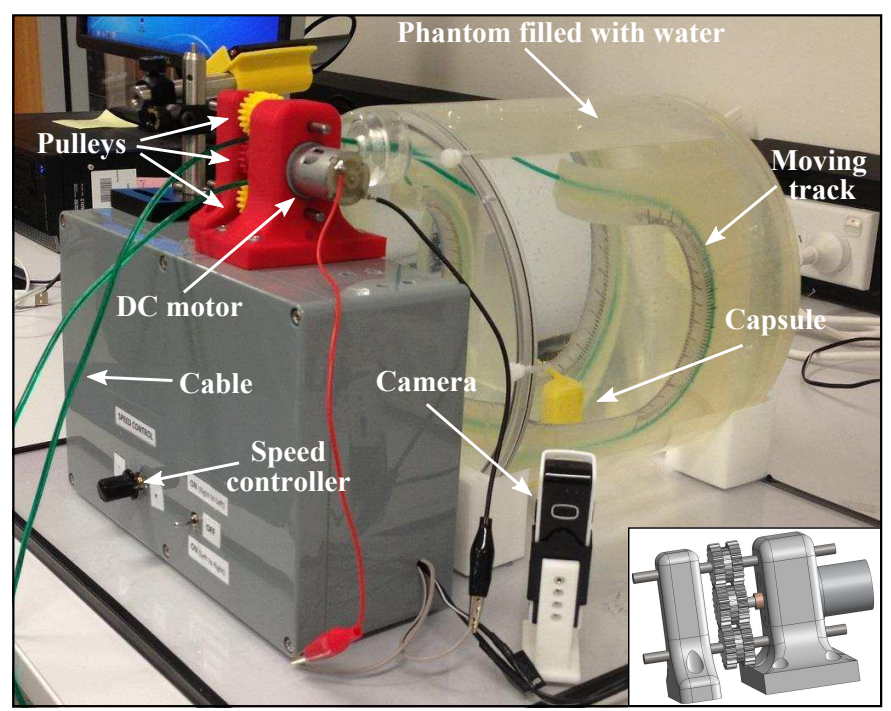

Fig. 6. Overview of the experimental apparatus (the 3D design model of the drive system is shown at the right bottom of the figure) 


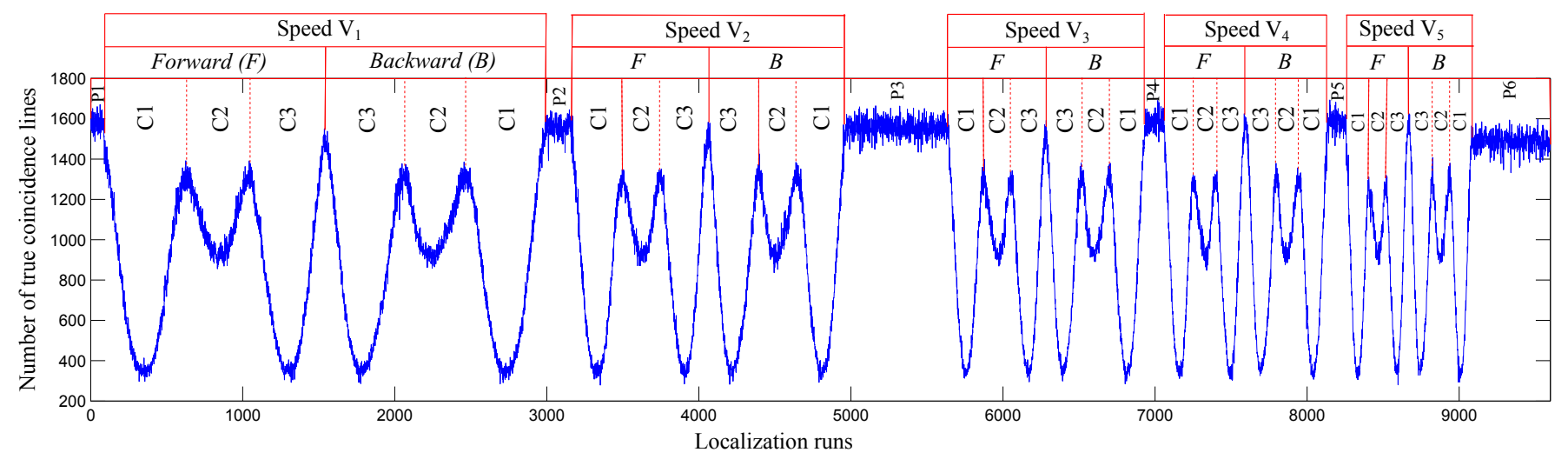

Fig. 8. Number of true coincidence lines obtained in each localization run (50ms) throughout 8 -minute scanning by a TF64 PET scanner. $V_{1}$ (7.5 mm/s), $V_{2}(12 \mathrm{~mm} / \mathrm{s}), V_{3}(17 \mathrm{~mm} / \mathrm{s}), V_{4}(21 \mathrm{~mm} / \mathrm{s})$, and $V_{5}(27 \mathrm{~mm} / \mathrm{s})$ are five different capsule speeds tested in the experiment. In each speed, the capsule moved forward (F) from one end to the other end of the track and returned (backward-B) before its speed was changed. C1, C2 and C3 are the regions corresponding to three different curves of the track that the capsule passed as mentioned in Subsection III-A3. P1 to P6 are six intended pauses when the capsule stopped moving and its speed was set to the next level.

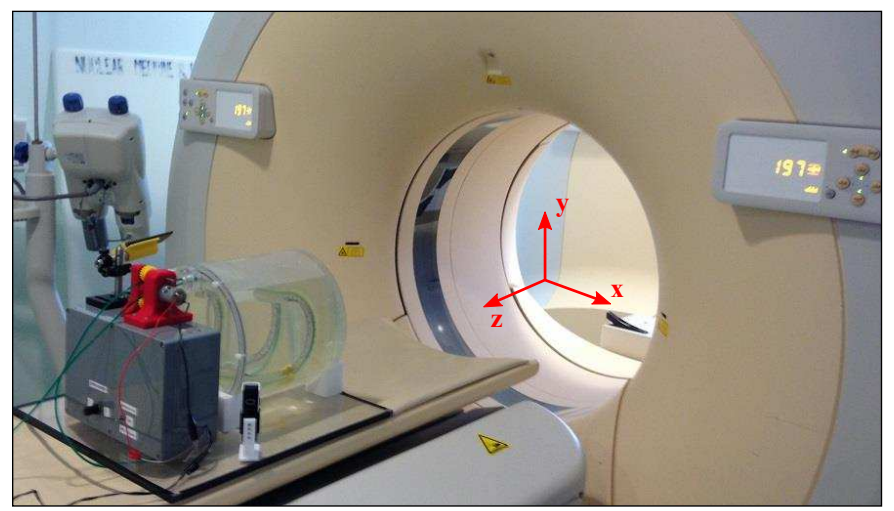

Fig. 7. The experimental apparatus was placed on the patient bed of a Philips TF64 PET scanner before being moved to the centroid for scanning

the main experiment started.

At the beginning of the calibration step, the marker holder was inserted into the track. One marker was then placed firmly on the holder. The actual position of the radioactive core of this marker was the origin of the local coordinate system of the track. A quick scan was performed so that the exact position of the marker could be determined. To ensure a high accuracy for this calibration step, the phantom was scanned without water to avoid scattered coincidences. It should be noted that the purpose of the calibration step was only to find the actual localization data of the capsule for later performance evaluation. For localization alone, it was unnecessary.

Once the calibration completed, the patient bed and the whole experimental apparatus were not allowed to move relatively in order to maintain the calibrated alignment between the two coordinate systems during the main experiment.

\section{Processing experimental data}

In the Philips PET system, there are several different acquisition modes. In order to extract the Cartesian coordinates of two ends of all coincidence lines, we used the Crystal Acquisition List Mode Format, in which the crystal row index and crystal number in the given row of the two crystals that have detected the coincidence gamma rays are stored. Based on the known geometry of the PET scanners, the list of pairs of index numbers were converted to a list of pairs of XYZ coordinates. The conversion process can be found in Appendix.

\section{RESULTS}

After the data obtained from the PET systems had been converted, they were input into the tracking algorithm for performance evaluation of the proposed localization method. Matlab (MathWorks, Inc.) was used in both the conversion process and the implementation of the tracking algorithm. Approximately 16.63 million coincidence lines were recorded in the first experiment ( 8 minute scanning by the TF64 scanner). In other words, there were on average 1,732 coincidence lines in each localization time interval (50 milliseconds). However, not all of the coincidence lines recorded in each run were used in the tracking algorithm. To maintain a low computational time without significantly affecting the tracking accuracy, a fraction of them is sufficient.

Throughout a total of 9,600 localization runs, the capsule passed through the three different sections $(\mathrm{C} 1, \mathrm{C} 2$ and $\mathrm{C} 3$ ) of the track several times as illustrated in Fig. 8. The axial position of the capsule with respect to the PET scanner varied during its journey along the track. Surti et al. [35] have shown that the system sensitivity of a PET Gemini TF scanner drops off linearly with increasing axial separation from the scanner's centroid. Therefore, as expected, the number of true coincidence lines reduced linearly when the capsule moved axially from the scanner's centroid to the axial extremes. Figure 8 presents the number of true coincidence lines obtained in each localization run. As can be seen from the figure, the number of true coincidence lines recorded at the middle of the curves $\mathrm{C} 1$ and C3 were lowest. This is because these points were placed furthest away from the scanner's centroid in the experiments.

Based on this sensitivity-related reason, we made an improvement to our previous work [28] in which a constant number of coincidence lines was used in every localization run. 
In this study, we input different number of coincidence lines to the tracking algorithm in each localization run. Depending on the sensitivity of the scanner at the previously estimated positions in the previous runs, the number of coincidence lines needed was calculated accordingly. This is because the axial positions of the capsule are assumed to be almost unchanged in two consecutive localization runs (50ms time difference). Regardless of the axial position of the capsule, this ensured that there were approximately 100 true coincidence lines per marker per run. In the first localization run, all recorded coincidence lines were used in the tracking algorithm. The estimate of initial values for the markers' position was thus more accurate.

Compared with the first experiment, only 11.02 million coincidence lines were recorded by the Allegro scanner in 8 minutes. This is understandable since TF64 is a newer PET scanner with higher resolution and detection efficiency. The minimum acquisition time frame in an Allegro scanner is $100 \mathrm{~ms}$, thus the length of a localization run for the second experiment was set at $100 \mathrm{~ms}$. Therefore there were in total of 4,800 localization runs in the second experiment and an average of 2,296 coincidence lines recorded in each localization run.

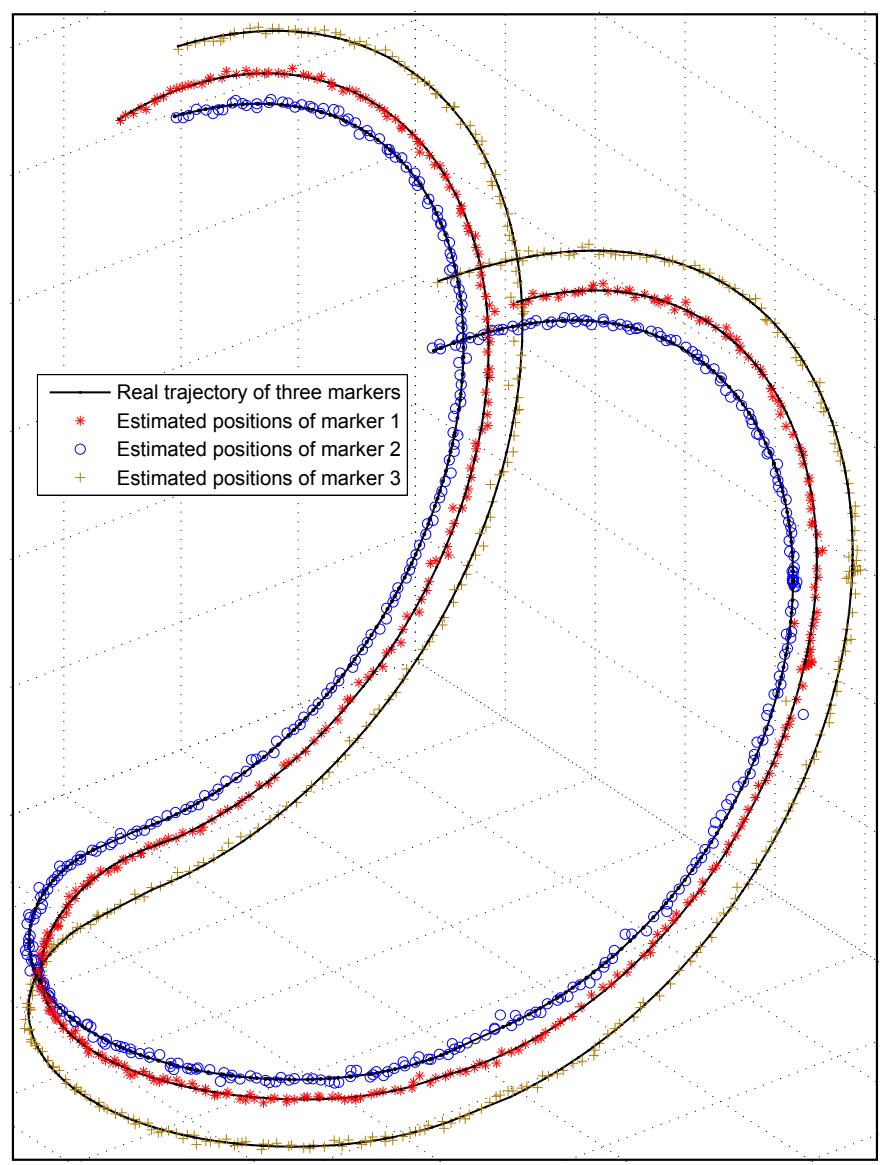

Fig. 9. 3D trajectories of estimated positions and actual movement trajectories of the three markers on the same figure for comparison. These trajectories were plotted based on the data obtained when the capsule moved with the highest speed of $27 \mathrm{~mm} / \mathrm{s}$.

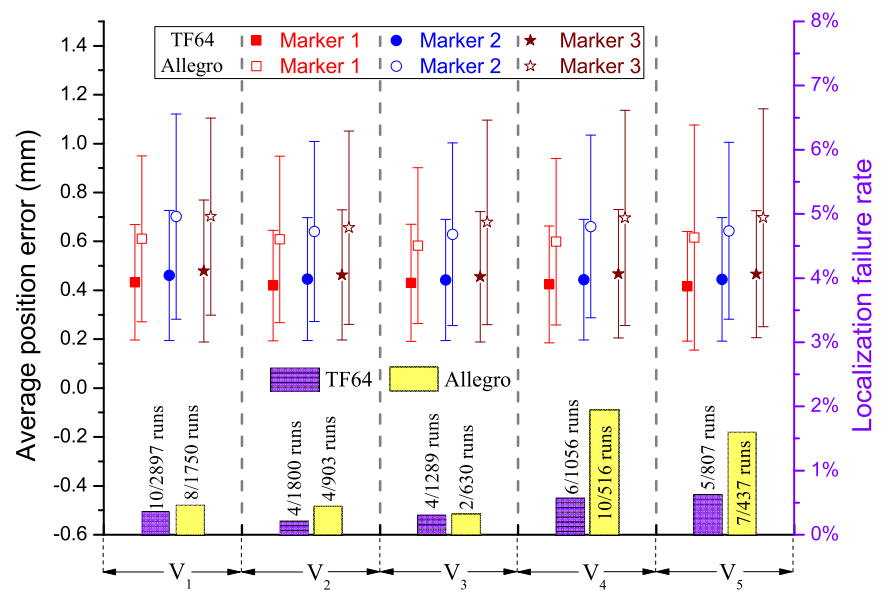

Fig. 10. Top plot: Average position error (mean \pm deviation) of each marker in different speed ranges of the capsule $\left(V_{1}\right.$ to $\left.V_{5}\right)$. Bottom plot: Failure rates of the localization in different speed ranges of the capsule. Data obtained from both scanners (TF64 and Allegro) are presented for performance comparison.

\section{A. Position error of each marker}

Over 9,600 localization runs (by TF64 scanner), the tracking algorithm successfully located the three markers in 9,571 runs $(99.7 \%$ success rate). The success rate in the second experiment (Allegro scanner) was 4,769/4,800 localization runs $(99.35 \%)$. In both experiments, the localization failed because the input data were composed of only two clusters instead of three. This occurred only at some extreme locations in the axial field-of-view (FOV) of the PET scanner which is explained in more detail in the Section V.

Figure 9 presents the 3D trajectories of estimated positions of the markers computed by the tracking algorithm, together with actual movement trajectories of the markers when the capsule moved with the highest speed of $27 \mathrm{~mm} / \mathrm{s}$. On average, the tracking algorithm took $6 \mathrm{~ms}$ to track the three markers in each localization run (computed by a $3.4 \mathrm{GHz}$ Intel Core i7 processor).

The position errors of the three markers in each localization run can be evaluated by comparing the estimated positions computed by the tracking algorithm with the true positions recorded during the experiments. As demonstrated by simulation in [28], the tracking algorithm is expected to achieve submillimeter accuracy. Therefore, the true positions of the markers need to be determined with a resolution of at least tens of micrometers every 50ms. Although the true markers' positions could be obtained by visualization using the engraved marks and three cameras as described in Subsection III-A3, these data were not precise enough to be used as reference data in such a short time interval. However, the actual trajectory of the capsule movement is known based on the design of the track. The position errors can thus be computed by fitting estimated data to the designed trajectory of the track.

Figure 10 shows average position errors of the three markers in each speed range of the capsule movement for both two experimental data sets by TF64 and Allegro scanners. The localization failure rate in each speed range is also presented in the figure. Through observation, the localization is considered 
a failure when the relative activity of at least one marker drops below 0.01 , or the relative distance between any two markers is less than $7 \mathrm{~mm}$ or larger than $16 \mathrm{~mm}$. This is because the markers would not be classified correctly into their corresponding clusters if these values are reached. In this case, the tracking algorithm stops and proceeds to the next localization run. As shown in Fig. 10, the average position error of each marker falls between $0.4 \mathrm{~mm}$ to $0.5 \mathrm{~mm}$ when TF64 scanner is used, while Allegro scanner provides approximately $0.6 \mathrm{~mm}$ to $0.7 \mathrm{~mm}$ average position error. Since the ${ }^{22} \mathrm{Na}$ marker that has highest activity (1.60Mbq) was chosen as Marker 1 (Fig. 4), the average position error of this marker is always smallest among the three markers as illustrated in Fig. 10.

\section{B. Orientation error of the capsule}

Based on the marker configuration in the capsule body as shown in Fig. 2, we can define a vector that originates from a midpoint between two closest markers to the furthest marker as the orientation vector of the capsule. A different angle between an estimated orientation vector (found by three estimated positions of the markers) and a fitted orientation vector (based on three fitted positions of the markers on the actual trajectory) is considered the orientation error of the capsule.

TABLE I

AVERAGE ORIENTATION ERROR OF THE CAPSULE IN DIFFERENT SPEED LEVELS OF THE CAPSULE MOVEMENT

\begin{tabular}{c|c|c} 
Capsule speed & TF64 scanner & Allegro scanner \\
\hline $\left.\mathbf{V}_{\mathbf{1}} \mathbf{( 7 . 5} \mathbf{~ m m} / \mathbf{s}\right)$ & $2.18^{\circ} \pm 1.19^{\circ}$ & $3.26^{\circ} \pm 1.77^{\circ}$ \\
$\mathbf{V}_{\mathbf{2}}(\mathbf{1 2} \mathbf{~ m m} / \mathbf{s})$ & $2.22^{\circ} \pm 1.21^{\circ}$ & $3.36^{\circ} \pm 1.79^{\circ}$ \\
$\left.\mathbf{V}_{\mathbf{3}} \mathbf{( 1 7} \mathbf{~ m m} / \mathbf{s}\right)$ & $2.21^{\circ} \pm 1.19^{\circ}$ & $3.35^{\circ} \pm 1.75^{\circ}$ \\
$\mathbf{V}_{\mathbf{4}}(\mathbf{2 1} \mathbf{~} \mathbf{~ m} / \mathbf{s})$ & $2.31^{\circ} \pm 1.21^{\circ}$ & $3.34^{\circ} \pm 1.83^{\circ}$ \\
$\left.\mathbf{V}_{\mathbf{5}} \mathbf{( 2 7} \mathbf{~} \mathbf{m m} / \mathbf{s}\right)$ & $2.32^{\circ} \pm 1.22^{\circ}$ & $3.46^{\circ} \pm 1.92^{\circ}$
\end{tabular}

The average orientation error of the capsule for each movement speed range of the capsule is described in Table I. As seen in the table, the average orientation error of the capsule was less than $2.4^{\circ}$ when the TF64 scanner was used, and was less than $3.5^{\circ}$ for data collected by the Allegro scanner.

\section{Relative distance error}

Another important parameter in the evaluation of the tracking performance is the relative distance between any pairs of the three estimated markers. For 9600 localization runs (by the TF64 scanner), these data were calculated and plotted in Fig. 11. The figure shows that the average relative distances between the three markers for the entire data set were $10.57 \pm 0.49 \mathrm{~mm}, 13.89 \pm 0.44 \mathrm{~mm}$ and $13.46 \pm 0.41 \mathrm{~mm}$, respectively. Although the designed relative distances are $10 \mathrm{~mm}, 13 \mathrm{~mm}, 13 \mathrm{~mm}$ as mentioned in Subsection II-B, due to manufacturing error the actual relative distances measured were a few hundred micrometers larger than the designed relative distances. Another source of errors between the calculated relative distances and the designed distances is the position error of each marker. In the second experiment (by

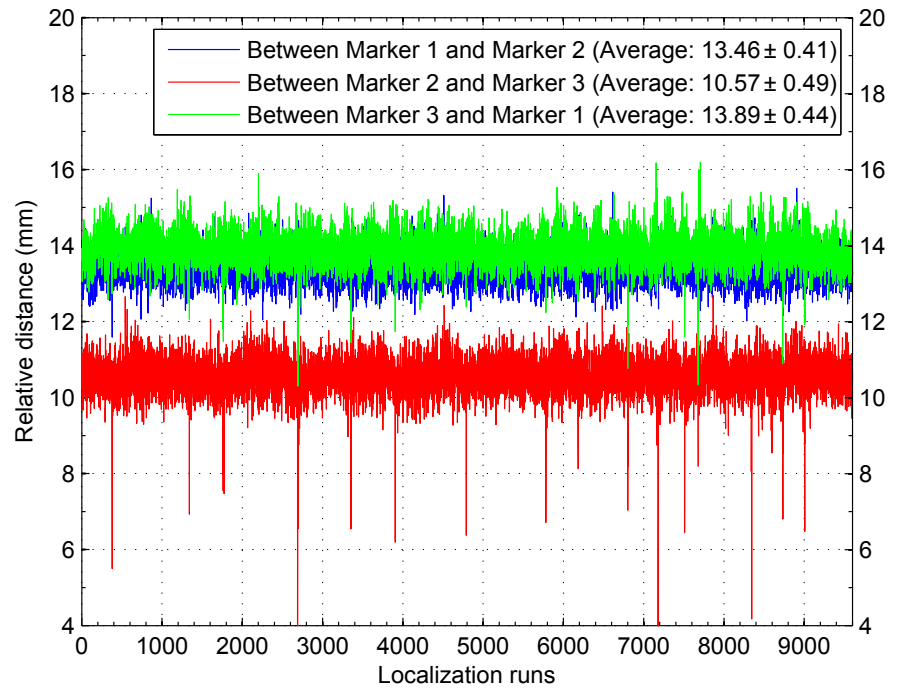

Fig. 11. The relative distance between any two of the three estimated markers when TF64 scanner was used. The red points that fall below the majority of data points indicate the failed localization runs.

the Allegro scanner), over 4800 localization runs, the average relative distances were $10.96 \pm 0.69 \mathrm{~mm}, 13.81 \pm 0.68 \mathrm{~mm}$ and $13.72 \pm 0.63 \mathrm{~mm}$, respectively.

\section{Precision of the tracking algorithm}

During the experiment, there were several periods of time when the capsule remained stationary at one location, such as when the capsule completed the entire track and stopped before moving with a new speed, or at the end of the experiment when all the speeds had been tested (Fig. 8). Although different sets of gamma rays were generated in each localization run, the markers could still be located at almost the same location. In order to evaluate the precision of the tracking algorithm, the variation and deviation in the position change of each marker for the localization runs in which the capsule remained stationary were assessed.

TABLE II

PRECISION OF THE TRACKING ALGORITHM BY EVALUATING THE POSITION CHANGE OF EACH MARKER IN THE PERIODS WHEN THE CAPSULE REMAINED STATIONARY AT ONE POSITION

\begin{tabular}{c|c|c|c|c|c}
\multirow{2}{*}{ Scanners } & \multirow{2}{*}{ Markers } & \multicolumn{2}{|c|}{ Deviation in XYZ components } & \multirow{2}{*}{$\begin{array}{c}\text { Distance to center } \\
\text { of mass (mm) }\end{array}$} \\
\cline { 3 - 5 } & & $\mathbf{X}(\mathbf{m m})$ & $\mathbf{Y}(\mathbf{m m})$ & $\mathbf{Z}(\mathbf{m m})$ & $0.44 \pm 0.19$ \\
\hline \multirow{3}{*}{ TF64 } & Marker 1 & 0.29 & 0.30 & 0.22 & $0.47 \pm 0.21$ \\
& Marker 2 & 0.32 & 0.32 & 0.25 & 0.47 \\
& Marker 3 & 0.30 & 0.27 & 0.24 & $0.43 \pm 0.19$ \\
\hline \multirow{3}{*}{ Allegro } & Marker 1 & 0.43 & 0.45 & 0.30 & $0.62 \pm 0.28$ \\
& Marker 2 & 0.53 & 0.52 & 0.44 & $0.78 \pm 0.37$ \\
& Marker 3 & 0.48 & 0.45 & 0.40 & $0.69 \pm 0.33$
\end{tabular}

The variation and deviation of a marker's position change over a period in which the marker remained stationary were calculated based on the distance from the estimated position of the marker in each localization run to the center of mass of all the estimated positions of the marker for all the localization runs in this period. The average and standard deviation of these distances for both experiments are shown in Table II. As 
seen in the table, these values are very close to the average position error mentioned in Subsection IV-A. In addition, the standard deviation of the position change in each of the $\mathrm{XYZ}$ components for each marker's estimated position is also included in Table II.

\section{DiscusSiON}

Although Fig. 10 shows that the average position error of each marker remains almost unchanged when the speed of the capsule movement varies from $7.5 \mathrm{~mm} / \mathrm{s}$ to $27 \mathrm{~mm} / \mathrm{s}$, this does not mean that the movement speed of the capsule has no effect on the performance of the tracking algorithm. By increasing the movement speed, the position change of the capsule in one localization time interval is increased. This would result in a larger position error in the direction along the track. However as explained above the position error was calculated by taking residual errors after fitting estimated data into the known trajectory based on the experimental design. By doing this, the position error along the track was unintentionally omitted. Experiments using a better method for determining the actual position of the capsule rather than visualization will be considered in a future work. Since the position error is expected to be less than $0.5 \mathrm{~mm}$, the method needs to have a resolution of tens of $\mu \mathrm{m}$ in order to provide accurate reference data for calculating absolute position error.

Despite the above limitation, the experimental results in Fig. 10 have demonstrated a high robustness of the tracking algorithm. The tracking algorithm was able to locate the three markers when the capsule moved with a high speed of $27 \mathrm{~mm} / \mathrm{s}$. In conjunction with the evaluation of the relative distance and the evaluation of the precision explained above, the position error based on the residual error of fitting estimated data to actual movement trajectories is considered acceptable.

The high robustness of the tracking algorithm is also demonstrated through the capability of realizing failed localizations and getting back on track in the succeeding localization run. Over the entire experimental data set, the localization failed in several localization runs, but it successfully located the three markers in the subsequent runs. It should be noted that the failure was not caused by the algorithm itself, but it was due to the inadequate input data. As explained in Section IV, the number of true coincidence lines recorded at the middles of the curves $\mathrm{C} 1$ and $\mathrm{C} 3$ were lowest as these points were placed furthest away from the scanner's centroid in the experiments. When the capsule reached this location, due to the marker configuration in the capsule, Marker 3 which has lowest activity, was closer to the axial extremes of the PET scanner than the other two markers. This resulted in a significant difference in the number of true coincidence lines originated from Marker 3 and those arising from the other two markers. The input data of the tracking algorithm in this localization run was merely composed of two clusters of true coincidence lines. Therefore, it is understandable that the tracking algorithm was unable to extract the three markers from the given input data.

The limitation of the localization method at the locations that are too close to the axial extremes of the PET scanner can be overcome by moving the patient bed to ensure the current estimated positions of the capsule are always in the axial FOV of the scanner. The subsequent calculation can then be compensated easily by adding the translation vector of the patient bed movement to the reference coordinate system.

In this study, the performance of the localization method was evaluated offline based on post-processed data. The tracking algorithm could not be implemented in real-time as soon as a sufficient number of coincidence lines were recorded in each localization time interval. This is understandable as the detector systems used in the experiments were clinical PET scanners. The firmware of these systems is legally not able to be modified or updated. However, the average computational time of the tracking algorithm in each localization run is approximately $6 \mathrm{~ms}$, compared with $50 \mathrm{~ms}$ sampling time. Therefore, the localization method has the potential to achieve real-time tracking when a custom detector system is built, instead of using clinical PET systems.

An advantage of the proposed custom localization method over the conventional PET imaging technique is that this method does not require the full-ring geometry of the detector system. This would significantly reduce the cost and the complexity of the method.

In our previous work [28], the performance of a smaller detector system with a reduced geometry has been evaluated using simulation data generated by the GATE toolkit. Similar tracking performances were delivered by both systems. In our future work, the tracking algorithm will be tested in a reduced geometry scanner with only two pairs of detector modules arranged at a given angle to each other. The sensitivity of the scanner with restricted geometry is expected to be significantly lower than that of a full-ring scanner. This will result in a lower number of coincidence lines recorded in each localization time interval. However, in this paper, the large number of coincidence lines recorded were intentionally not all used in the implementation of the tracking algorithm in order to make it similar to what is expected when a smaller detector system is used. Therefore, the activity of the marker in the future experiments with two pairs of gamma detector blocks is not expected to differ greatly from the activity level chosen in this study.

\section{CONCLUSION}

This paper has presented an experimental evaluation of a novel localization method for a robotic endoscopic capsule based on tracking three position emission markers embedded in the cover of an endoscopic capsule. Using experimental data obtained from a Philips Allegro PET scanner and a Philips TF64 PET scanner, the performance of the proposed localization method has been evaluated. The results show that this method can potentially achieve real-time tracking with an average position error of less than $0.5 \mathrm{~mm}$ and average orientation error of less than $2.4^{\circ}$. Other important advantages of this method are that it does not occupy any space inside the capsule, it does not consume any power from the built-in battery, and that it can be compatible with magnetic actuation systems for controlling the capsule movement. One disadvantage of this localization method is the use of expensive PET 


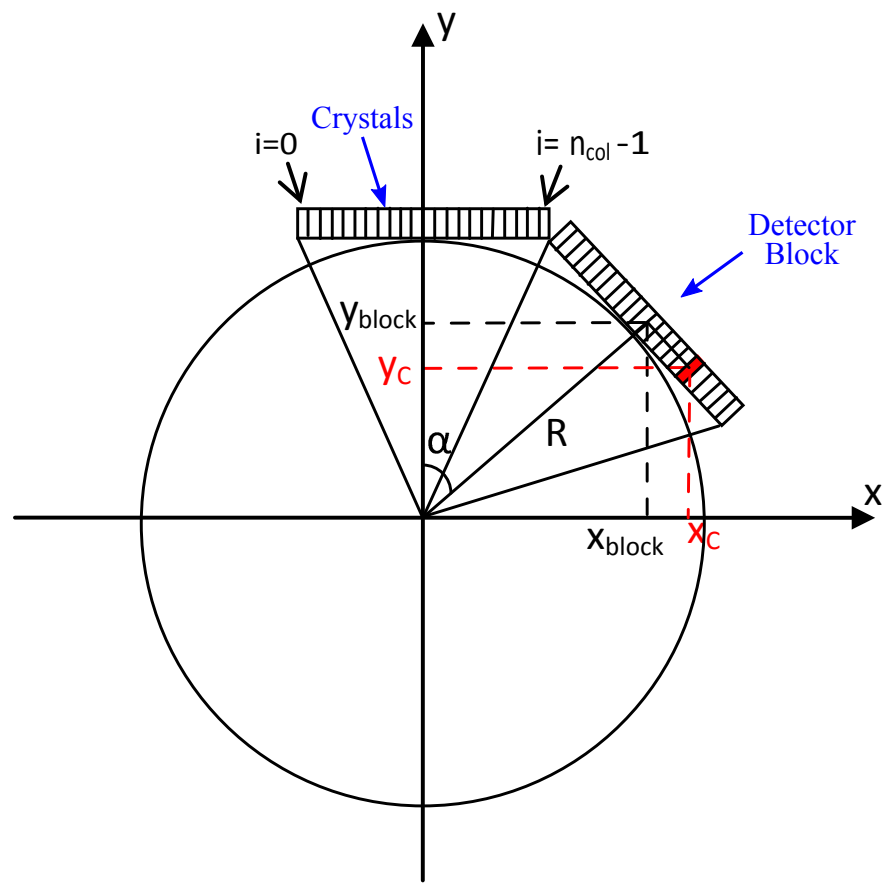

Fig. 12. A sketch of a PET scanner for conversion of crystal indices to XYZ coordinates. The rectangle in solid red pattern is the crystal being converted

scanning equipment. However, this method does not require the full-ring geometry of conventional PET scanners which is generally used for 3D imaging purposes. Instead, two pairs of detector modules in a fixed angle to each other would produce similar results. In our future work, we plan to establish a gamma-ray detection system based on the optimally reduced detector geometry, and subsequently demonstrate the efficacy of the proposed method using a larger phantom and a 3D model of the GI tract.

\section{APPENDIX}

\section{DATA CONVERSION TO CARTESIAN COORDINATES}

In this section, the conversion of crystal indices, stored in the Crystal Acquisition List Mode Format, to XYZ coordinates [36] is described.

For each coincidence line, four indices are stored, which are $\left(X_{a}, Z_{a}\right)$ and $\left(X_{b}, Z_{b}\right)$. Where $Z_{a}, Z_{b}$ are the row numbers and $X_{a}, X_{b}$ are the crystal numbers in the given row $Z_{a}, Z_{b}$ of the two crystals that have detected the coincidence gamma rays, respectively. Depending on the version of the Philips PET scanner, the full-ring scanner consists of $n_{\text {block }}$ detector blocks, each with $n_{\text {col }} \times n_{\text {row }}$ crystals. Therefore, the scanner has $n_{\text {row }}$ rows in the axial direction, and there are $\left(n_{\text {block }} \cdot n_{c o l}\right)$ crystals in each row. $Z_{a}, Z_{b}$ must be within 0 to $\left(n_{\text {row }}-1\right)$, and $X_{a}, X_{b}$ must be within 0 to $\left(n_{b l o c k} \cdot n_{c o l}-1\right)$

Obtained from the main header of the list-mode file, the crystal pitch in the axial direction is $d_{\text {pitch }}(\mathrm{mm})$. Since we have chosen the origin of the coordinate system is at the centroid of the scanner, the z-coordinate of a crystal $\left(X_{c}, Z_{c}\right)$ is given as below

$$
z_{c}=d_{\text {pitch }}\left(Z_{c}-\left(n_{\text {row }}-1\right) / 2\right)
$$

The angle between any two adjacent blocks seen from the centroid of the scanner is

$$
\theta=2 \pi / n_{\text {block }}
$$

The index of the block that contains the crystal is

$$
i_{\text {block }}=\left(X_{c} \operatorname{div} n_{c o l}\right)+1
$$

The index of the crystal in $\left(i_{\text {block }}\right)^{t h}$ block is

$$
i_{\text {crystal }}=X_{c} \bmod n_{c o l}
$$

The angle between $y$-axis and the line connecting the origin of the coordinate system and the center of $\left(i_{\text {block }}\right)^{\text {th }}$ block is

$$
\alpha=\left(i_{\text {block }}-1\right) \theta
$$

Given the crystal depth $d_{\text {depth }}(\mathrm{mm})$ and the radius of the scanner $R(\mathrm{~mm})$, the $\mathrm{x}$ and $\mathrm{y}$ coordinates of the center of $\left(i_{\text {block }}\right)^{t h}$ block is

$$
\begin{aligned}
& x_{\text {block }}=\left(R+d_{\text {depth }} / 2\right) \sin \alpha \\
& y_{\text {block }}=\left(R+d_{\text {depth }} / 2\right) \cos \alpha
\end{aligned}
$$

Given the crystal width $d_{w i d t h}$, distance from the crystal to the center of $\left(i_{\text {block }}\right)^{\text {th }}$ block is

$$
h=d_{\text {width }}\left(i_{\text {crystal }}-\left(n_{\text {col }}-1\right) / 2\right)
$$

Therefore, the $\mathrm{x}$ and $\mathrm{y}$ coordinates of the crystal (Fig. 12) is

$$
\begin{aligned}
& x_{c}=\left(R+d_{\text {depth }} / 2\right) \sin \alpha+h \cos \alpha \\
& y_{c}=\left(R+d_{\text {depth }} / 2\right) \cos \alpha-h \sin \alpha
\end{aligned}
$$

\section{ACKNOWLEDGMENT}

The authors would like to thank staff at the Department of Nuclear Medicine in Nepean Hospital, Sydney, Australia and the Department of Nuclear Medicine and Centre for PET in Austin Health Hospital, Melbourne, Australia for their invaluable help during the experiments.

\section{REFERENCES}

[1] A. Koulaouzidis, E. Rondonotti, and A. Karargyris, "Small-bowel capsule endoscopy: a ten-point contemporary review," World J. Gastroenterol., vol. 19, no. 24, pp. 3726-46, 2013.

[2] G. Iddan, G. Meron, A. Glukhovsky, and P. Swain, "Wireless capsule endoscopy," Nature, vol. 405, no. 6785, pp. 417-417, 2000.

[3] U. Kopylov and E. G. Seidman, "Clinical applications of small bowel capsule endoscopy," Clin Exp Gastroenterol, vol. 6, pp. 129-37, 2013.

[4] B. J. Nelson, I. K. Kaliakatsos, and J. J. Abbott, "Microrobots for minimally invasive medicine," Annu. Rev. Biomed. Eng., vol. 12, pp. $55-85,2010$.

[5] J. L. Toennies, G. Tortora, M. Simi, P. Valdastri, and R. J. Webster, "Swallowable medical devices for diagnosis and surgery: the state of the art," Proc. Inst. Mech. Eng. C J. Mech. Eng. Sci., vol. 224, no. 7, pp. 1397-1414, 2010.

[6] K. Kong, D. Jeon, S. Yim, and S. Choi, "A robotic biopsy device for capsule endoscopy," J. Med. Devices, vol. 6, no. 3, pp. 031004-031012, 2012.

[7] S. P. Woods and T. G. Constandinou, "Wireless capsule endoscope for targeted drug delivery: Mechanics and design considerations," IEEE Trans. Biomed. Eng., vol. 60, no. 4, pp. 945-953, 2013.

[8] K. Pahlavan, G. Bao, Y. Ye, S. Makarov, U. Khan, P. Swar, D. Cave, A. Karellas, P. Krishnamurthy, and K. Sayrafian, "Rf localization for wireless video capsule endoscopy," Int. J. Wireless Inform. Network, vol. 19, no. 4, pp. 326-340, 2012. 
[9] T. D. Than, G. Alici, H. Zhou, and W. Li, "A review of localization systems for robotic endoscopic capsules," IEEE Trans. Biomed. Eng., vol. 59, no. 9, pp. 2387-2399, 2012.

[10] D. Fischer, R. Schreiber, D. Levi, and R. Eliakim, "Capsule endoscopy: the localization system," Gastrointest. Endosc. Clin. N. Am., vol. 14, no. 1, pp. 25-31, 2004.

[11] P. Brida and J. Machaj, "A novel enhanced positioning trilateration algorithm implemented for medical implant in-body localization," Int. J. Antenn. Propag, vol. 2013, p. 10, 2013.

[12] A. Wille, M. Broll, and S. Winter, "Phase difference based rfid navigation for medical applications," in Proc. IEEE Int. Conf. Radio Freq. Identif., 2011, pp. 98-105.

[13] H. Chao, L. Mao, S. Shuang, Y. Wan'an, Z. Rui, and M. Q. H. Meng, "A cubic 3-axis magnetic sensor array for wirelessly tracking magnet position and orientation," IEEE Sens. J., vol. 10, no. 5, pp. 903-913, 2010.

[14] W. Weitschies, H. Blume, and H. Monnikes, "Magnetic marker monitoring: high resolution real-time tracking of oral solid dosage forms in the gastrointestinal tract," Eur. J. Pharm. Biopharm., vol. 74, no. 1, pp. 93-101, 2010

[15] F. Carpi and H. Shaheed, "Grand challenges in magnetic capsule endoscopy," Expert Rev. Med. Devices, vol. 10, no. 4, pp. 433-6, 2013.

[16] J. Keller, C. Fibbe, U. Rosien, and P. Layer, "Recent advances in capsule endoscopy: development of maneuverable capsules," Expert Rev Gastroenterol Hepatol, vol. 6, no. 5, pp. 561-6, 2012.

[17] X. Wang and M. Q. H. Meng, "Perspective of active capsule endoscope: actuation and localisation," Int. J. Mech. Autom, vol. 1, no. 1, pp. 38-45, 2011.

[18] M.-G. Kim, Y.-S. Hong, and E.-J. Lim, "Position and orientation detection of capsule endoscopes in spiral motion," Int. J. Precis. Eng. Manuf., vol. 11, no. 1, pp. 31-37, 2010

[19] M. Salerno, G. Ciuti, G. Lucarini, R. Rizzo, P. Valdastri, A. Menciassi, A. Landi, and P. Dario, "A discrete-time localization method for capsule endoscopy based on on-board magnetic sensing," Meas. Sci. Technol, vol. 23, no. 1, pp. 015 701-015710, 2012.

[20] C. Di Natali, M. Beccani, and P. Valdastri, "Real-time pose detection for magnetic medical devices," IEEE Trans. Magn., vol. 49, no. 7, pp. 3524-3527, 2013.

[21] Y. Sehyuk and M. Sitti, "3-d localization method for a magnetically actuated soft capsule endoscope and its applications," IEEE Trans. Robot., vol. 29, no. 5, pp. 1139-1151, 2013.

[22] K. M. Popek, A. W. Mahoney, and J. J. Abbott, "Localization method for a magnetic capsule endoscope propelled by a rotating magnetic dipole field," in Proc. IEEE Int. Conf. Robot. Autom, 2013, pp. 5348-5353.

[23] M. R. Basar, F. Malek, K. M. Juni, M. S. Idris, and M. I. M. Saleh, "Ingestible wireless capsule technology: A review of development and future indication," Int. J. Antenn. Propag, vol. 2012, pp. 1-14, 2012.

[24] E. Spyrou and D. K. Iakovidis, "Video-based measurements for wireless capsule endoscope tracking," Meas. Sci. Technol, vol. 25, no. 1, p. 015002, 2014

[25] Z. Nagy, M. Fluckiger, O. Ergeneman, S. Pane, M. Probst, and B. J. Nelson, "A wireless acoustic emitter for passive localization in liquids," in Proc. IEEE Int. Conf. Robot. Autom, 2009, pp. 2593-2598.

[26] J. J. Gumprecht, T. Lueth, and M. Khamesee, "Navigation of a robotic capsule endoscope with a novel ultrasound tracking system," Microsyst Technol, vol. 19, no. 9-10, pp. 1415-1423, 2013.

[27] A. Krieger, I. I. Iordachita, P. Guion, A. K. Singh, A. Kaushal, C. Menard, P. A. Pinto, K. Camphausen, G. Fichtinger, and L. L. Whitcomb, "An mri-compatible robotic system with hybrid tracking for mri-guided prostate intervention," Biomedical Engineering, IEEE Transactions on, vol. 58, no. 11, pp. 3049-3060, 2011.

[28] T. D. Than, G. Alici, S. Harvey, H. Zhou, and W. Li, "Concept and simulation study of a novel localization method for robotic endoscopic capsules using multiple positron emission markers," Med. Phys., vol. 41, no. 7, 2014.

[29] D. J. Parker, C. J. Broadbent, P. Fowles, M. R. Hawkesworth, and P. McNeil, "Positron emission particle tracking - a technique for studying flow within engineering equipment," Nucl. Instr. Meth. Phys. Res. A, vol. 326, no. 3, pp. 592-607, 1993.

[30] G. Saha, Basics of PET Imaging: Physics, Chemistry, and Regulations. Springer, 2010.

[31] T. Xu, J. T. Wong, P. M. Shikhaliev, J. L. Ducote, M. S. Al-Ghazi, and S. Molloi, "Real-time tumor tracking using implanted positron emission markers: Concept and simulation study," Med. Phys., vol. 33, no. 7, pp. 2598-2609, 2006.
[32] N. W. Churchill, M. Chamberland, and T. Xu, "Algorithm and simulation for real-time positron emission based tumor tracking using a linear fiducial marker," Med. Phys., vol. 36, no. 5, pp. 1576-1586, 2009.

[33] M. Chamberland, R. Wassenaar, B. Spencer, and T. Xu, "Performance evaluation of real-time motion tracking using positron emission fiducial markers," Med. Phys., vol. 38, no. 2, pp. 810-819, 2011.

[34] J. C. Bezdek, Pattern Recognition with Fuzzy Objective Function Algorithms. Kluwer Academic Publishers, 1981.

[35] S. Surti, A. Kuhn, M. E. Werner, A. E. Perkins, J. Kolthammer, and J. S. Karp, "Performance of philips gemini tf pet/ct scanner with special consideration for its time-of-flight imaging capabilities," J. Nucl. Med., vol. 48, no. 3, pp. 471-80, 2007.

[36] M. Chamberland, "Performance evaluation and algorithm development for real-time tumour tracking using positron emission markers," Ph.D. dissertation, Carleton University, 2009.

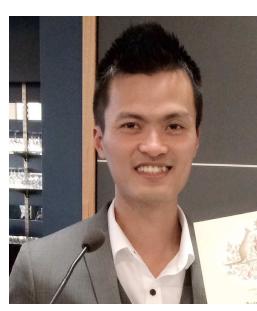

Trung Duc Than received the B.Eng. degree in Industrial Automation Engineering from the Hanoi University of Science and Technology, Hanoi, Vietnam, in 2007, and the M.Eng. degree in Electrical and Computer Engineering from the University of Wollongong, Wollongong, N.S.W, Australia, in 2010, where he is currently working toward the $\mathrm{Ph} . \mathrm{D}$. degree in the School of Mechanical, Materials, and Mechatronic Engineering.

His research interests include the development of localization and control systems for robotic applica-

tions.

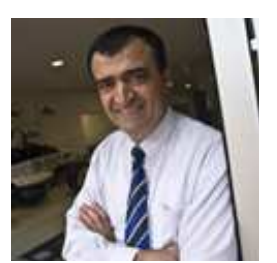

Gursel Alici received the Ph.D. degree in robotics from the Department of Engineering Science, Oxford University, Oxford, U.K., in 1994.

$\mathrm{He}$ is currently a Professor at the University of Wollongong, Wollongong, Australia, where he is the Head of the School of Mechanical, Materials and Mechatronic Engineering. His current research interests are soft robotics, system dynamics and control, robotic drug delivery systems, novel actuation concepts for biomechatronic applications, robotic mechanisms and manipulation systems, soft and smart actuators and sensors, and medical robotics. He has published more than 250 refereed publications in his areas of research.

Dr. Alici was a Technical Editor of the IEEE/ASME TRANSACTIONS ON MECHATRONICS during 20082012. He is a Technical Editor of the IEEE Access, the first IEEE open access journal with interdisciplinary scope. He is a Member of the Mechatronics National Panel formed by the Institution of Engineers, Australia. He has served on the international program committee of numerous IEEE/ASME International Conferences on Robotics and Mechatronics. He was the General Chair of the 2013 IEEE/ASME International Conference on Advanced Intelligent Mechatronics held in Wollongong, Australia. He is the leader of Soft Robotics for Prosthetic Devices theme of the ARC Center of Excellence for Electromaterials Science. He received the Outstanding Contributions to Teaching and Learning Award in 2010 and the 2013 Vice-Chancellors Interdisciplinary Research Excellence Award from the University of Wollongong. 


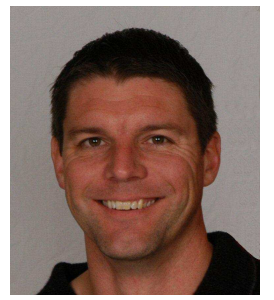

Steven Harvey received his $\mathrm{PhD}$ degree in Biomedical Engineering from the University of Aberdeen, UK in 1999 as a Commonwealth Scholar. He also holds BE and ME degrees in Mechanical Engineering from the University of Wollongong, NSW, Australia. He currently manages the Department of Nuclear Medicine at Wollongong Hospital, NSW, Australia.

His research interests include non-invasive imaging of the GI tract, PET/CT imaging, load-bearing spinal MRI imaging, 3D visualisation + additive manufacture in orthopaedics, and radiation dosimetry. Dr Harvey has published numerous articles in peer-reviewed medical and technical journals. He has over 15 years of experience working in the medical imaging sector in both engineering and physics capacities. He is accredited by the Australasian College of Physical Scientists and Engineers in Medicine in the field of Nuclear Medicine Physics, and is registered as a Certified Professional Engineer with Engineers Australia.

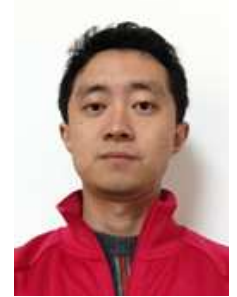

Hao Zhou received the B.Eng. degree in Building Environment and Facility Engineering from Tongji University, Shanghai, China in 2004. He received the M.Eng. degree in Mechanical Engineering from The University of Queensland, Queensland, Australia in 2008. He received his second M.Eng. degree in Engineering Practice (Mechanical) from University of Wollongong, New South Walse, Australia in 2009. $\mathrm{He}$ is currently working towards the Ph.D. degree at University of Wollongong, New South Walse, Australia.

His research interests include simulations and analysis of electromagnetics, simulations and analysis of biomechanics of small intestine, mechanics of viscoelastic materials, and active locomotion of wireless capsule endoscopy.

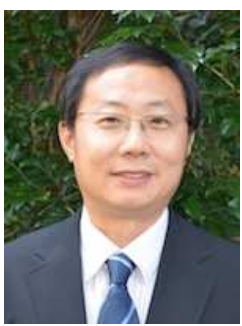

Weihua Li received the B.Eng. and M.Eng. degrees from the University of Science and Technology of China, Hefei, China, in 1992 and 1995, respectively, and the Ph.D. degree from Nanyang Technological University (NTU), Singapore, in 2001.

After two years postdoctoral training in NTU, he joined the University of Wollongong, Wollongong, N.S.W., Australia, as a Full-Time Academic, where he is currently working as a Discipline Advisor for Mechatronic Engineering. His research interests include smart materials and structures, microuidics, intelligent mechatronics, and dynamics and vibration control. He has coauthored more than 160 articles, and delivered many plenary or invited talks at various international conferences. He is currently serving as Editorial Board Member for more than eight international journals.

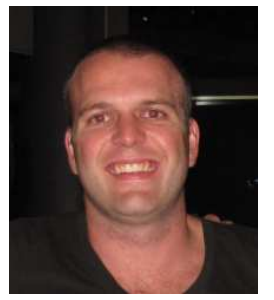

Trent Cook completed his BAppSc(Nuclear Medicine) degree from the University of Sydney in 1998. He is currently employed as the Senior Nuclear Medicine Technologist at the Nuclear Medicine and PET department at Nepean Hospital, Penrith N.S.W, Australia.

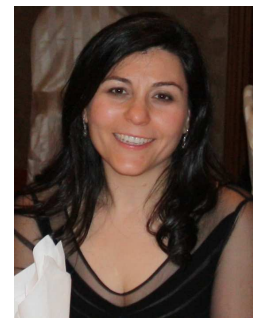

Sharon Alam-Fotias received her B.S. degree in Medical Radiation Technology (Nuclear Medicine) from the University of Sydney, Australia in 1999. She currently is the Chief Medical Radiation Scientist and Head of Department for the Nuclear Medicine and PET Department at Nepean Hospital. She is also a fully qualified CT radiographer in Diagnostic imaging.

Her research interests include PET/CT imaging, PET tracers and radiation dosimetry in PET and Nuclear Medicine. She is currently involved in a number of research projects within the department as well as multicentre and multination trails. Over her 20 years of working she has published articles in peer-reviewed medical journals as well as presented and at a number of National and International conferences. She is accredited by the Australian and New Zealand Society of Nuclear Medicine and is registered and Certified by the Medical Radiation Practise Board of Australia. 